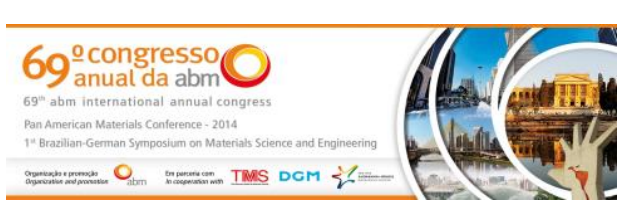

Tema: Iniciação Científico-tecnológica

\title{
SIMULAÇÃO COMPUTACIONAL DO PROCESSO DE TREFILAÇÃO AVALIANDO POSSÍVEIS CAUSAS DE DISTORÇÕES NA PRODUÇÃO DE BARRAS AISI 1045*
}

\author{
Rafael Paini Pavlak ${ }^{1}$ \\ Juliana Zottis² \\ Alexandre da Silva Rocha ${ }^{3}$
}

\section{Resumo}

A trefilação a frio para produção de barras de aço é realizada em função da sua precisão dimensional e modificação das propriedades mecânicas do material. $\mathrm{O}$ processo consiste na conformação de um fio-máquina que, guiado por um puxador, passa através de uma ferramenta (fieira de trefilação). Tal procedimento irá inserir tensões internas na barra chamadas tensões residuais, as quais, dependendo da sua distribuição e intensidade, podem causar distorções do produto final e possivelmente seu descarte. A fim de prever o comportamento destas tensões foram desenvolvidas simulações tridimensionais no software Deform ${ }^{\mathrm{TM}}$. Este trabalho teve por objetivo explorar os efeitos do desalinhamento angular da fieira combinado com uma heterogeneidade na distribuição do atrito, avaliando a influência na distribuição de tensões residuais na barra trefilada. Observou-se certa influência na distribuição das tensões finais, atingindo diferenças em torno de $300 \mathrm{MPa}$ entre superfícies, 0 que caracteriza um potencial de distorções no produto final.

Palavras-chave: Simulação; Trefilação a frio; Distorções; Tensões residuais.

\section{COMPUTATIONAL SIMULATION OF THE COLD DRAWING PROCESS TO EVALUATE THE POSSIBLE CAUSES OF DISTORTIONS IN STEEL BARS AISI 1045 PRODUCTION}

\section{Abstract}

The cold drawing process is used to produce steel bars with dimensional precision and modification of mechanical properties, as strength, that it is possible to reach in a high speed. This metalworking process uses tensile forces to stretch metal, where the wirerod is drawn through a die to reduce its diameter and increase its length. The process of reducing the cross sectional area of wire, bar or tube by drawing the material through a die without any pre-heating. The process changes the mechanical properties of the steel and the finished product is accurate to size, free from scale with a bright surface finish. This process will generate internal stresses in the bar; they are called residual stresses, that, depending the intensity and distribution, they may cause distortions in the final product and perhaps the disposal of the bars. In order to predict the stresses behavior, was developed some tridimentional simulations with Deform ${ }^{\mathrm{TM}}$ simulation software. These work had main objective to study the effects of angular misalignments of the die combining with the heterogeneity distribution of the friction coefficient, studding their influence in the final stresses distributions. Might be observed some influence in the final stresses distributions, with differences in order of $300 \mathrm{Mpa}$ comparing the surfaces, featuring a potential of distortions in the final product.

Keywords: Simulation; Cold drawing; Distortions; Residual stresses.

1 Graduando de Engenharia Mecânica, LdTM, UFRGS, Campus do Vale, Porto Alegre, RS, Brasil; paini.pavlak@ufrgs.br.

2 Engenheira Mecânica, Mestra, LdTM, UFRGS, Porto Alegre, RS, Brasil; juzottis@hotmail.com.

3 Engenheiro Mecânico, Prof. Dr. Pesquisador, LdTM, UFRGS, Porto Alegre, RS, Brasil; alexandre.rocha@ufrgs.br.

\footnotetext{
* Contribuição técnica ao 69 Congresso Anual da ABM - Internacional e ao 14ํㅡㄹ ENEMET - Encontro Nacional de Estudantes de Engenharia Metalúrgica, de Materiais e de Minas, 21 a 25 de julho de 2014, São Paulo, SP, Brasil.
} 


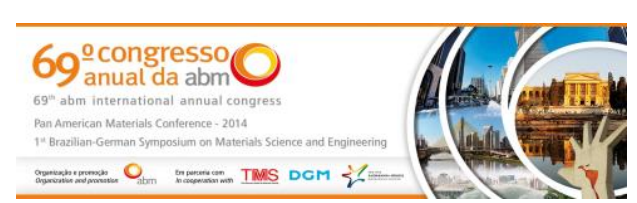

\section{INTRODUÇÃO}

O emprego da simulação computacional utilizando o método de elementos finitos, ao longo dos últimos anos, vem ganhando atenção devido às vantagens oferecidas. Muitas empresas continuam empregando o método de "tentativa e erro" quando estão iniciando uma nova produção; esse método, além de não ser muito eficaz, pode ocasionar perdas no processo e elevação dos custos. Através da simulação é possível analisar as falhas do processo, evitar perdas futuras em energia e material, aperfeiçoar a qualidade e melhorar a competitividade do produto final [1,2].

Sabendo dessas vantagens, nos últimos anos foram realizadas inúmeras simulações de conformação mecânica. Em especial a trefilação é um dos processos que é muito visado por várias indústrias para a fabricação de arames, barras cilíndricas, entre outros, devido à precisão dimensional e melhora das propriedades do material [3].

O processo de trefilação consiste basicamente em tracionar matéria-prima através de uma ferramenta cônica chamada fieira, onde a força de tração é exercida por garras que se fixam na extremidade da barra. À medida que o material vai sendo trefilado, ele sofre deformação plástica resultando em uma redução da área da seção transversal. Grandes vantagens do processo de trefilação são o excelente acabamento superficial e a alta velocidade produtiva do processo [4].

A Figura 1 mostra os componentes principais necessários ao processo de trefilação.

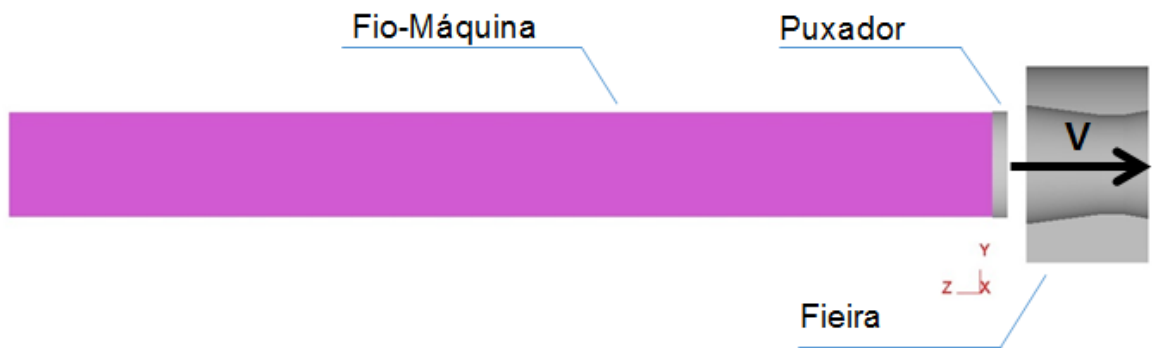

Figura 1. Unidades presentes no processo de trefilação.

Pelo fato deste processo de trefilação causar deformações no material próximas ao limite de conformabilidade, ou seja, quando a tensão de escoamento é muito próxima da tensão limite de resistência; ocorre o fenômeno metalúrgico chamado encruamento ou endurecimento. As consequências decorrentes desse fenômeno são a redução da ductilidade e aumento da resistência mecânica do material [4].

Após o processo de trefilação, pelo fato de campos de deformações plásticas não uniformes terem atuado no material, a barra final apresentará tensões residuais. Elas existem em praticamente todas as peças rígidas, metálicas ou não. Não é possível diferenciar peças com ou isentas de tensões residuais pelas suas aparências. Entretanto, as características físicas e mecânicas das duas podem ser diferentes [5]. No caso das barras trefiladas, essas tensões fornecem um aumento na resistência mecânica que é fundamental para a utilidade dessas barras que, neste caso estudado, é servir para a produção de eixos automobilísticos.

Entretanto, a maneira com que o equipamento de trefilação é montado propicia para a presença de desalinhamentos e lubrificações não uniformes [4]. O processo é dependente da influência do coeficiente de atrito, devido aos estresses inseridos e também a energia necessária para que o material passe pela fieira.

Portanto, este trabalho visa o estudo da influência do desalinhamento da fieira combinado com a heterogeneidade da distribuição do atrito na precisão e qualidade do produto final após o processo de trefilação de barras de aço AISI 1045.

\footnotetext{
* Contribuição técnica ao $69^{\circ}$ Congresso Anual da ABM - Internacional e ao 14ํㅡㄹ ENEMET - Encontro Nacional de Estudantes de Engenharia Metalúrgica, de Materiais e de Minas, 21 a 25 de julho de 2014, São Paulo, SP, Brasil.
} 


\section{MATERIAIS E MÉTODOS}

O material em estudo é o aço AISI $1045 \mathrm{com}$ as suas propriedades à temperatura ambiente, pois o processo é realizado a frio. Pela sua composição de $0,45 \%$ de carbono, é considerado um aço de médio teor de carbono; apresenta boas propriedades mecânicas e tenacidade. As simulações foram realizadas considerando o material isotrópico, com propriedades elasto-plásticas e não foram inseridas propriedades de tocas de calor. As ferramentas foram consideradas rígidas; o puxador tem uma geometria de um pequeno cilindro com um raio um pouco maior que o da barra, tendo a finalidade de prender-se a barra pela sua extremidade e tracioná-la através da fieira.

Os parâmetros de entrada dos modelos de simulação já foram estudados e utilizados por trabalhos de Zottis [4] e Soares [3]. Os principais parâmetros utilizados para a análise e simulação do processo são apresentados na Tabela 1 e Figura 2.

Tabela 1. Parâmetros do processo de trefilação

\begin{tabular}{l|l}
\hline Material & AISI 1045 \\
\hline Temperatura & $20^{\circ} \mathrm{C}$ \\
\hline Atrito entre Fio Máquina e Fieira $(\mu)$ & 0,1 e 0,15 Coulomb \\
\hline Velocidade de trefilação & $1250 \mathrm{~mm} / \mathrm{s}$ \\
\hline Diâmetro inicial e final & $21,46 \mathrm{~mm}$ e 20,25 mm \\
\hline Redução da área transversal da barra & $11 \%$ \\
\hline Ângulo de entrada da fieira $(2 \alpha)$ & $15^{\circ}$ \\
\hline Módulo de Young (E) & $210 \mathrm{GPa}$ \\
\hline Coeficiente de Poisson $(v)$ & 0.3 \\
\hline Tensão de escoamento inicial & $390 \mathrm{MPa}$ \\
\hline Curva de escoamento & $k_{f}=1292,8 . \varphi^{0,2018}$ \\
\hline
\end{tabular}

Representação da curva de escoamento equação Ludwik-Hollomon obtida através da curva tensão $x$ deformação do material (Figura 2).

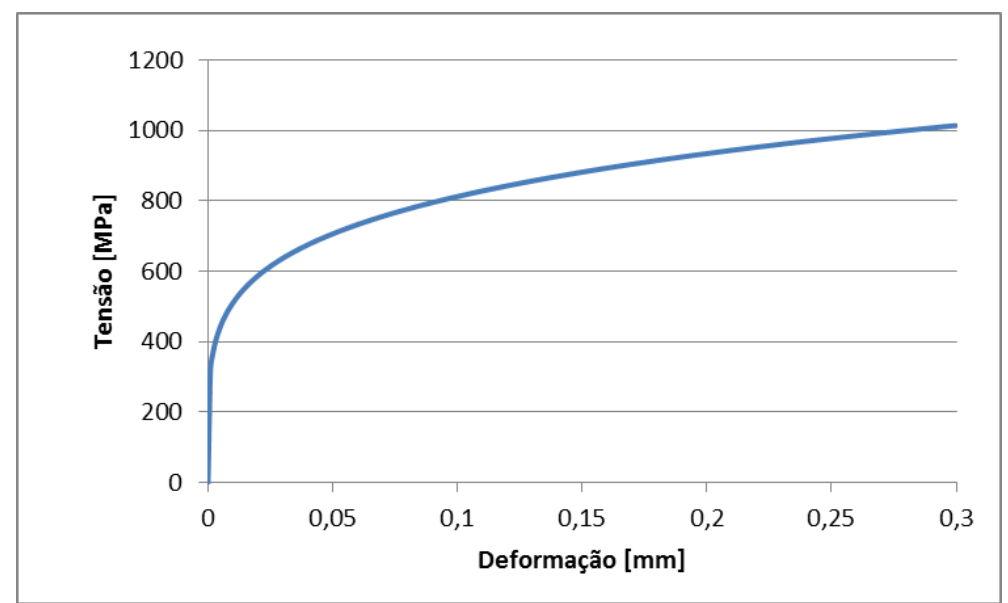

Figura 2. Curva tensão $x$ deformação do material.

Os componentes (barra, fieira e puxador) da simulação foram desenhados em 2D e convertidos para o 3D através de uma rotação em torno do eixo de simetria. Esse processo permite criar uma malha hexaédrica axissimétrica que, segundo Soares [3], possibilita uma melhor análise das tensões residuais e das distorções pelo fato de apresentar elementos homogêneos e bem distribuídos.

\footnotetext{
* Contribuição técnica ao $69^{\circ}$ Congresso Anual da ABM - Internacional e ao 14ํㅡㄹ ENEMET - Encontro Nacional de Estudantes de Engenharia Metalúrgica, de Materiais e de Minas, 21 a 25 de julho de 2014, São Paulo, SP, Brasil.
} 

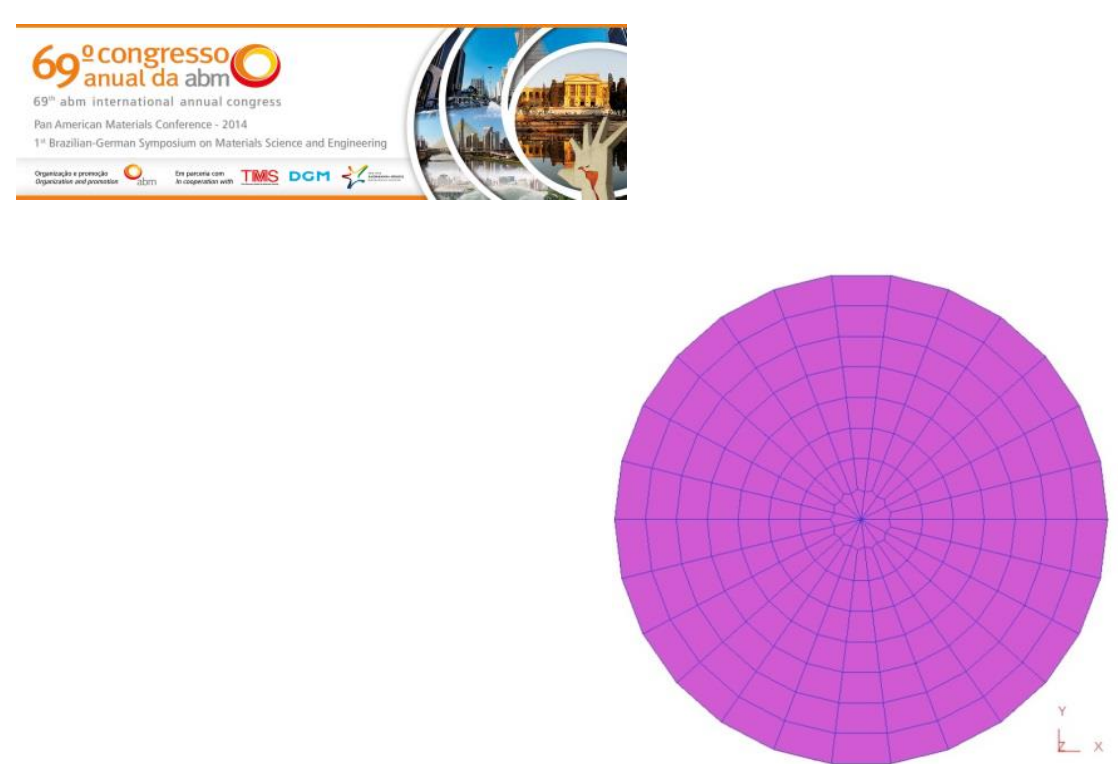

Figura 3. Malha hexaédrica do fio máquina.

$\mathrm{Na}$ Figura 4 está representado o desalinhamento do eixo da fieira, sendo que esta foi rotacionada em $1,5^{\circ}$ em torno do ponto $P$ e em sentido anti-horário. A barra tem comprimento de $200 \mathrm{~mm}$, raio de $10,73 \mathrm{~mm}$. A fieira possui ângulo de entrada de $15^{\circ}$ e raio de saída de $10,125 \mathrm{~mm}$. A dimensão do ângulo na figura foi aumentada para facilitar entendimento.

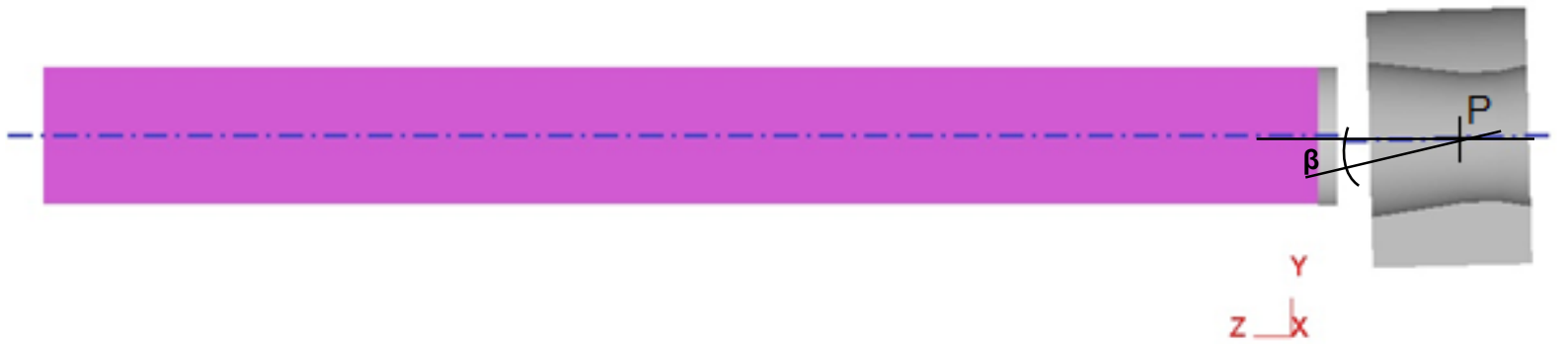

Figura 4. Perfil das simulações com a fieira desalinhada.

O valor de $1,5^{\circ}$ visa estudar o que um possível erro no momento de montagem do equipamento de trefilação possa mudar no perfil da barra final, como tensões residuais não uniformes, deformações excessivas e defeito geométrico. Os resultados podem ser comparados com estudos anteriores, havendo a possibilidade de observar as consequências de ângulos menores de desalinhamento.

$\mathrm{O}$ atrito tem influência direta no acabamento da peça e no desgaste da ferramenta, além de exigir maior esforço de trefilação e aumentar a temperatura de trabalho [6]. Por consequência, foram estudadas as consequências da falta de homogeneidade no coeficiente de atrito entre a barra e a fieira. Este fator foi estudado separadamente e em conjunto com o desalinhamento citado acima. A Figura 5 exemplifica como foram elaborados os modelos com lubrificações não homogêneas. Dentro da região demarcada foi inserido coeficiente de atrito de 0,15 Coulomb e na outra metade da fieira 0 atrito foi de 0,1 Coulomb. $O$ coeficiente de atrito para processos de trefilação de fio máquinas com pequenas deformações tem um valor em torno de 0,1 Coulomb [7].

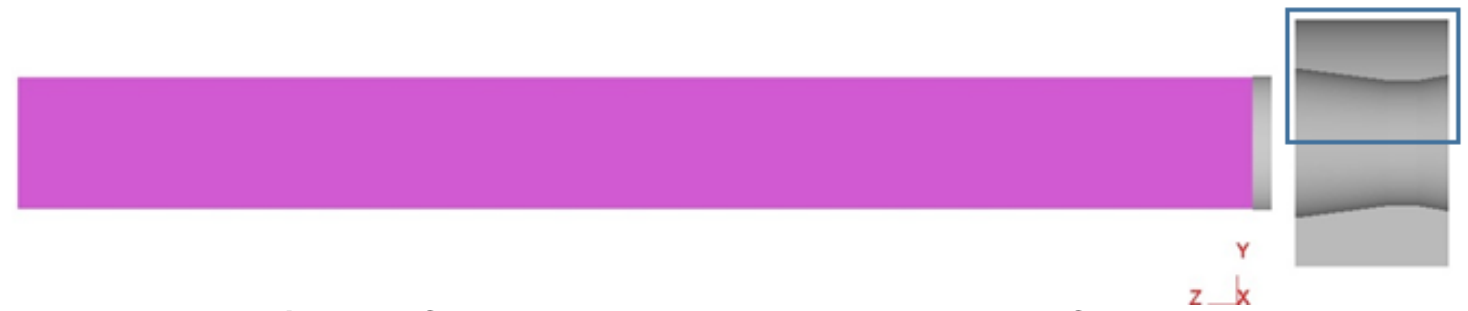

Figura 5. Caixa demarcando a região de atrito 0,15 Coulomb.

\footnotetext{
* Contribuição técnica ao $69^{\circ}$ Congresso Anual da ABM - Internacional e ao 14ํㅡㄹ ENEMET - Encontro Nacional de Estudantes de Engenharia Metalúrgica, de Materiais e de Minas, 21 a 25 de julho de 2014, São Paulo, SP, Brasil.
} 


\subsection{Tensões Residuais}

A Figura 8 mostra o perfil das tensões residuais na direção axial dos modelos simulados, bem como quais parâmetros foram inseridos para cada um. O primeiro foi chamado de simulação modelo, pois ele representa o processo de trefilação no seu caso ideal, sem a presença de qualquer tipo de desalinhamento e com lubrificação homogênea ao longo da barra de 0,1 Coulomb.

Tabela 2. Características de cada simulação

\begin{tabular}{ccc}
\hline Nome & Presença de diferentes atritos & Fieira desalinhada \\
\hline Modelo & - & $0^{\circ}$ \\
\hline Caso 2 & 0,15 Coulomb na metade $+Y$ & $0^{\circ}$ \\
\hline Caso 3 & 0,15 Coulomb na metade $+Y$ & $1,5^{\circ}$ \\
\hline Caso 4 & 0,15 Coulomb na metade $-Y$ & $1,5^{\circ}$ \\
\hline Caso 5 & - & $1,5^{\circ}$ \\
\hline
\end{tabular}
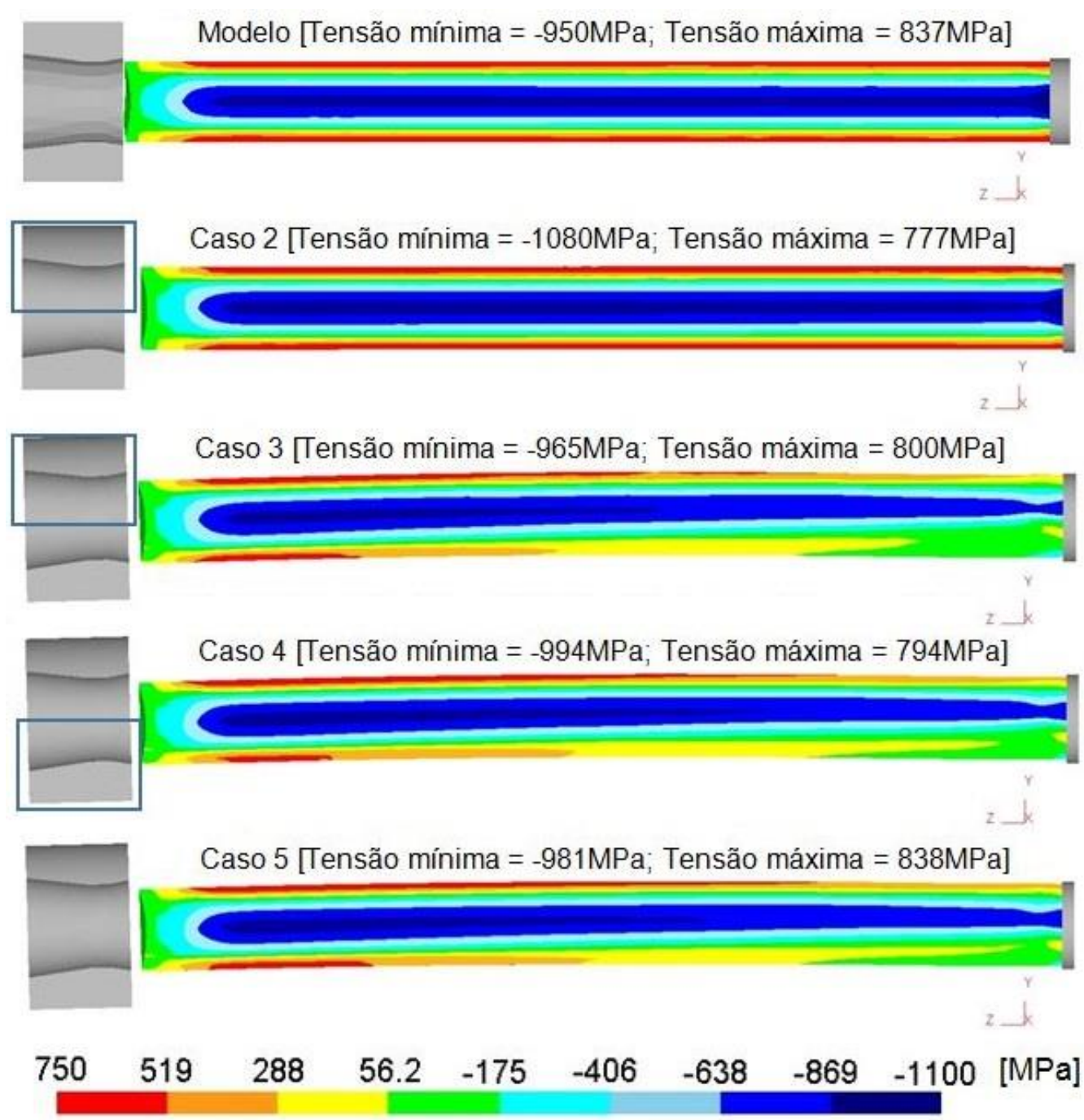

Figura 8. Perfis de Tensões Residuais na Direção Axial de Trefilação (Z).

Analisando os perfis resultantes da simulação numérica, verifica-se que a distribuição das tensões residuais ao longo da barra varia tanto na superfície quanto no centro, sendo que os valores mais críticos estão situados no lado oposto ao desalinhamento da fieira.

* Contribuição técnica ao $69^{\circ}$ Congresso Anual da ABM - Internacional e ao 14ํㅡㄹ ENEMET - Encontro Nacional de Estudantes de Engenharia Metalúrgica, de Materiais e de Minas, 21 a 25 de julho de 2014, São Paulo, SP, Brasil. 


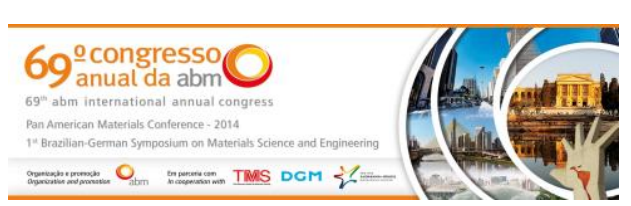

Tabela 3. Resultantes das simulações

\begin{tabular}{lccccc}
\hline & Modelos & Caso 2 & Caso 3 & Caso 4 & Caso 5 \\
\hline Deformação & 0,12473 & 0,12856 & 0,13032 & 0,12961 & 0,126151 \\
\hline Taxa de deformação & 38,2591 & 37,71260 & 40,583 & 41,146 & 48,806 \\
\hline Força [N] & 73361,56 & 80640,93 & 81831,09 & 82760,89 & 74940,22 \\
\hline
\end{tabular}

Pode-se observar que a força necessária exercida pelo puxador para que a trefilação ocorra é acrescida de maneira significativa, aproximadamente $7 \mathrm{kN}$, quando a lubrificação da fieira não for homogênea. $O$ fato de a força de trefilação aumentar por causa da má lubrificação pode acarretar um maior desgaste das peças, causar o aparecimento de defeitos superficiais e ainda aumentar o trabalho necessário para o funcionamento do processo [6].

Uma maneira de contornar este problema da lubrificação é sugerida por Utsunomiya [11] em sua produção a utilização de uma fieira com superfície porosa tem por objetivo aumentar a ação do fluído lubrificante e consequentemente sua eficácia.

\section{CONCLUSÃo}

Este trabalho teve por objetivo o estudo de duas possíveis causas com influência no aparecimento de distorções após processo de trefilação do aço AISI 1045: o desalinhamento angular na montagem da fieira e a falta de homogeneidade do atrito entre a fieira e o fio-máquina.

Conforme o que foi apresentado, quando há o desalinhamento da fieira, a heterogeneidade no perfil de tensões residuais pode resultar em um agravante das distorções que vem aparecendo após este processo de trefilação estudado. Esse pequeno desalinhamento de $1,5^{\circ}$ tem grande chance de ocorrer uma vez que as unidades de trefilação são todas ajustadas e montadas manualmente, permitindo ou facilitando erros de ajustes destes equipamentos.

Com o foco no estudo das consequências da lubrificação não homogênea, evidencia-se uma influência maior na resultante da força de trefilação. Fato que além de aumentar a energia necessária para possibilitar o processo, também colabora para o desgaste tanto de peça quanto de ferramenta.

\section{Agradecimentos}

Agradecemos a Capes, CNPq e FINEP pelo apoio financeiro através do programa Bragecrim (Brazilian German Collaborative Research Initiative in Manufacturing Technology) e também a FAPERGS por financiar uma bolsa de iniciação científica.

\section{REFERÊNCIAS}

1 Schaeffer L, Brito AMG, Geier M. Numerical Simulation Using Finite Elements to Develop and Optimize Forging Process. 2005; 2(3): 199-200

2 Tekkaya AE. Stat-of-the-Art of Simulation of Sheet Metal Forming" Journal of Material Processing Technology, 2000; 103:14-22.

3 Soares CAT. Análise das Tensões Residuais no Processo de Trefilação Considerando os Efeitos de Anisotropia [Mestrado]. Porto Alegre: UFRGS, 2012.

4 Zottis J. Modelagem e Análise Numérica na Previsão de Distorções no Processo de Trefilação de Barras de Aço [Master Teses - Programa de Pós Graduação em

\footnotetext{
* Contribuição técnica ao 69 Congresso Anual da ABM - Internacional e ao 14ํㅡㄹ ENEMET - Encontro Nacional de Estudantes de Engenharia Metalúrgica, de Materiais e de Minas, 21 a 25 de julho de 2014, São Paulo, SP, Brasil.
} 
Engenharia de Minas, Metalúrgica e de Materiais - PPGE3M]. Porto Alegre: UFRGS, 2013.

5 Carmo DC. Análise de Tensões por Ultra-Som em Barra de Aço Sob Flexão [Mestrado]. Rio de Janeiro: UFRJ; 2006.

6 Palmeira AA. Capítulo 5: Processos de Trefilação. 2005 [acesso em 30 de Janeiro de 2014]; 18-22. Disponível em:

http://www.fat.uerj.br/intranet/disciplinas/Processos\%20de\%20Fabricacao\%20IV/Cap\% 205\%20-\%20Trefila\%E7\%E3o.pdf

7 Schaeffer L. Conformação Mecânica. 2 ed. Porto Alegre. Imprensa Livre Editora; 2004.

8 SFCT. Manual do Usuário. Deform ${ }^{\mathrm{TM}}$. ANO: 50-51

9 Rulz J, Atienza JM, Elices M. Residual Stresses in Wires: Influence of Wire Length. Journal of Material Engineering and Performance. CASM International, 2006;12: 480489.

10 Nunes RM. Estudo de Distorção de Barras Cilíndricas de Aço ABNT 1045 em uma Rota de Fabricação Envolvendo Trefilação Combinada e Têmpera por Indução [PhD Teses - Programa de Pós Graduação em Engenharia de Minas, Metalúrgica e de Materiais - PPGE3M]. Porto Alegre: UFRGS; 2012.

11 Utsunomiya $H$, Takagishi S, Ito A, Matsumoto R. Lubrication Using Porous Surface Layer for Cold Drawing of Steel Wire. ScienceDirect, 2013; 0007-8506.

* Contribuição técnica ao 69 Congresso Anual da ABM - Internacional e ao 14ํㅡㄹ ENEMET - Encontro Nacional de Estudantes de Engenharia Metalúrgica, de Materiais e de Minas, 21 a 25 de julho de 2014, São Paulo, SP, Brasil. 